\title{
Agent-based Modeling and Simulation for Target-centric Warfare
}

\author{
Xiong $\mathrm{Li}^{\mathrm{a}}$, Wei Pu, Zhanning Han \\ Department of Command and Administration \\ Academy of Armored Force Engineering, 100072, Beijing \\ alixiong2609@126.com
}

\begin{abstract}
Keywords: Agent, Agent-based Modeling and Simulation, Target-centric Warfare.
Abstract. Target-centric warfare is a new warfare mode and means with various intelligent entities that form an adaptive loop. Based on analysis on the process of target-centric warfare, agent and multi-agent system technology is used and agent-based target-centric warfare models including interaction protocol model and behavior simulation model are designed. Thus, agent-based target-centric warfare simulation is implemented. The feasibility and efficiency of the proposed approach is validated by case study of Red-Blue Forces counterwork.
\end{abstract}

\section{Introduction}

With the evolution of technology, the methods for warfare have also changed. These methods would also very likely depend on military technologies. In modern wars, commanders should have the initiative by various information devices, make decision and command promptly. Therefore, the need of building the fast and exact warfare loop has come to the fore. It is time to think about changing the name of network-centric warfare to "target-centric warfare". The use of the term network-centric insinuates that it is the network that is central to the successful prosecution of the warfare. However, the term target-centric implies that the warfare loop is enabling the synchronization of warfare, which is the realm of the commander. In fact, this study has shown that it is the effective use of information by individual commanders and the effective interactions of those commanders that enabled the successful prosecution of the war [1].

The development of intelligent simulation system technology has also changed the way we think about warfare. Diversified warfare platforms, e.g., tanks, are usually distributed, in which updates are made asynchronously and resources come interactive without centralized control. In order to demonstrate these warfare platforms and resources dynamically in a computer system, without military risks, we need a reasonable intelligent simulation system [2].

Agent and multi-agent system emerged, as a scientific area, from the previous research efforts in distributed artificial intelligence started in 1980s. Agents are an important area of research in the sense that they are proactive, and include: goal-directed and communication capabilities. Furthermore, each goals of the agent are constantly changing in a dynamic environment [3]. Agent-based simulation paradigm has become a useful tool in analyzing complex processes and intelligent systems $[2,3,4,5,6,7,8]$. Thus, it can be viewed as a feasible method to explore target-centric warfare. In this paper, we design an agent-based simulation model to demonstrate some warfare loop activities.

\section{Target-centric Warfare Analysis}

The network centric approach to warfare is the military embodiment of information age concepts. Studies have shown that networking enables forces to undertake a different range of missions than non-networked forces, by improving both efficiency and effectiveness of operations. Network-centric warfare uses computers and communications to link warfare platforms and resources through information flows that depend on the interoperability of systems. Network-centric warfare involves collaboration and sharing of information to ensure that all appropriate assets can be quickly brought to 
bear by commanders during combat operations [9]. From the view of command and control, the process of network-centric warfare can be shown as Fig. 1.

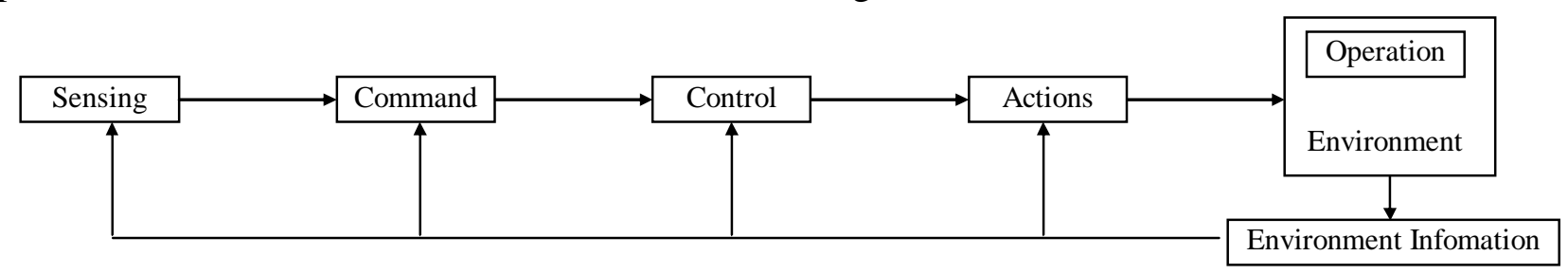

Fig.1 Process of network-centric warfare.

The analysis and comprehensive understanding of warfare loop activities is extremely difficult and in most cases impossible for humans to grasp without the assistance of advanced tool, since there are some new needs, such as: 1) command posts must have a plan to train and integrate diversified resources into an organization with high warfare capability; 2) the warfare loop requires flexibility to quickly adjust processes and structural design to maximize the sharing of information and increased collaboration in varying situations [1]; 3) the warfare loop requires all actions to be pointed to targets. Thus, target-centric warfare has become an important warfare mode and means in modern wars. The new warfare mode and means has an innovative process, i.e., an adptive loop, shown as Fig. 2.

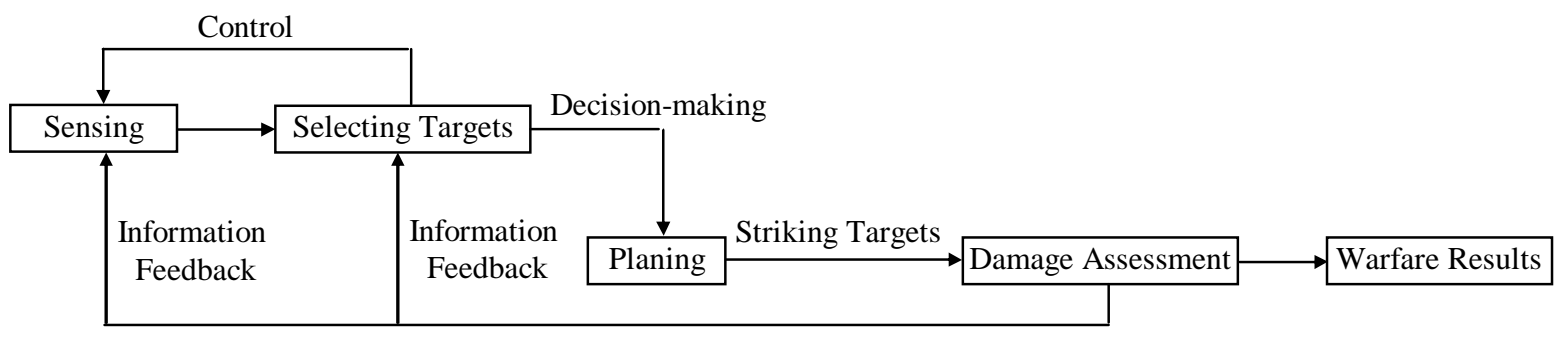

Fig.2 Process of target-centric warfare.

From the above figures, we can find that target-centric warfare roots in network-centric warfare. They all emphasize improved understanding of the operational situation at all levels of command, and increased ability to tap into the collective knowledge of all forces to reduce the "fog and friction" commonly referred to in descriptions of fighting [9]. However, there are some differences. Network-centric warfare emphasizes self-synchronization, or doing what needs to be done without traditional orders, but target-centric warfare emphasizes decision-making, command and control for warfare actions to pursue the most damage to the selected targets, and thus forms a target-centric warfare loop.

\section{Agent-based Models Design and Case Study}

Agent-based modeling and simulation technology gives concept developers a view to the future by enabling them to simulate and manipulate in near real-time, the assets and operational conditions. Agent-based modeling and simulation is an intuitive method to study intelligent systems as it allows researchers to include an array of details in their simulation [5]. Agents are autonomous, flexible and proactive. In addition, agents have multi-threaded control and can build a control to act only if they want to $[2,3,4,5,6,7,8]$. Agent is an active entity and chooses a decision process dynamically through interactive with environment. The agent makes a choice from a large set of possibilities which can determine the transformation of environmental information, the internal information and decision process. Thus the result is the mode of operation that an agent choose [8]. The entities of target-centric warfare loop run with autonomy and interaction. These information interactions include sending, accepting, and informing all kinds of command and control instruction, striking selected targets based on navigation information, measurement intelligence, and surveillance information, and assessing the damage to the striked taegets. 


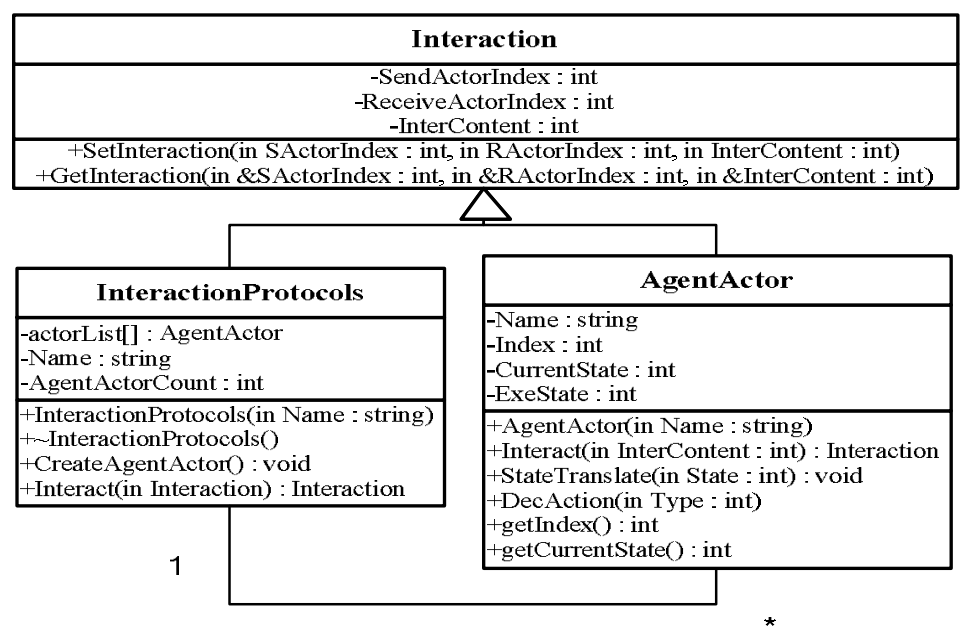

Fig.3 Function class in the interaction protocol model.

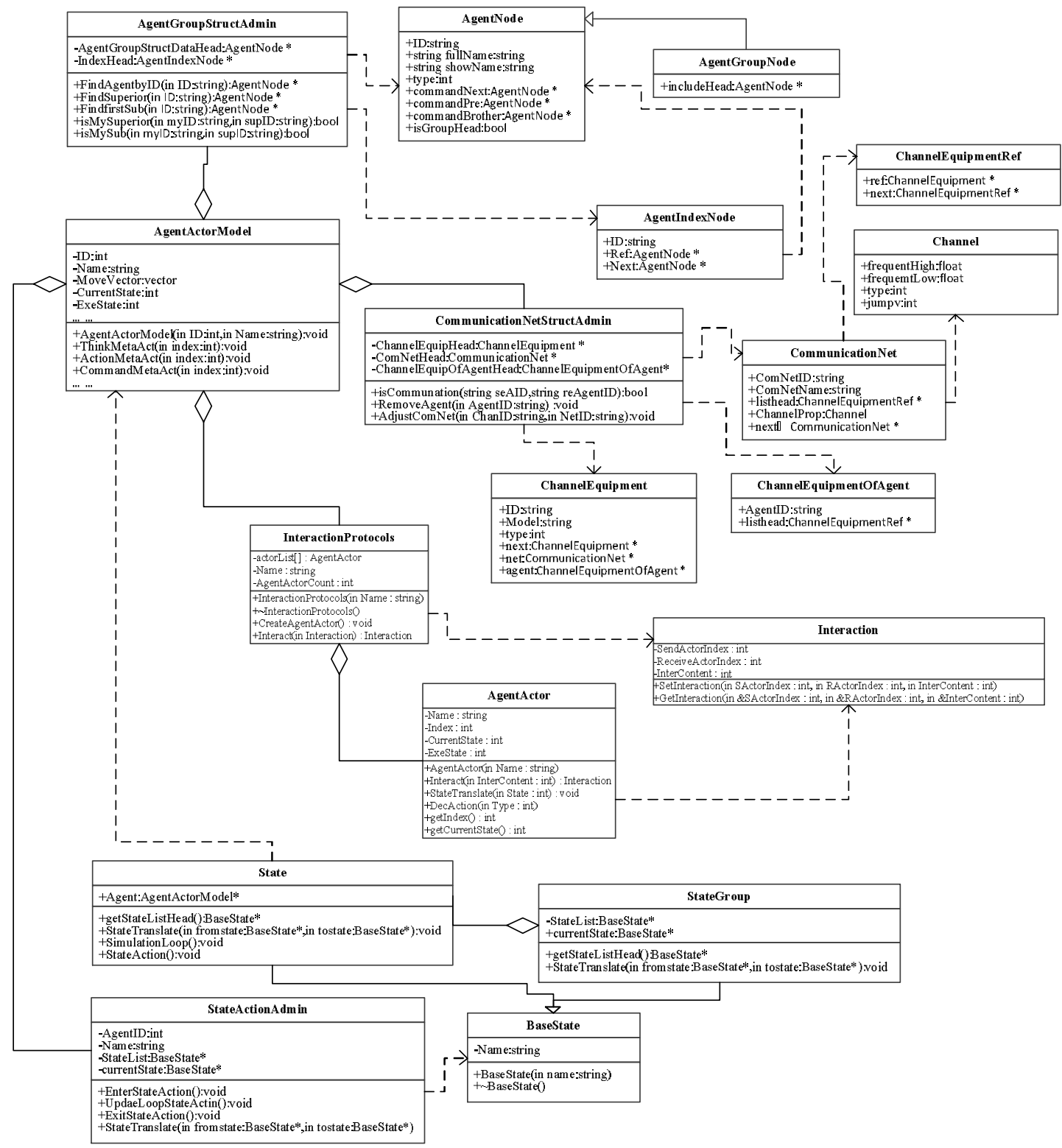

Fig.4 Behavior simulation model framework class.

Target-centric warfare system is so alike a distributed multi-agent system in behaviors that we can set up mappings from its entities to respective agents. The key to design multi-agent interaction protocol model is analysis of target-centric warfare system including organization and entities. We can 
define the function class in this interaction protocol model as Fig. 3. The behavior simulation model framework class can be designed as Fig. 4.

In this paper, we take Red-Blue Forces counterwork into account and give some experiment parameters in target-centric warfare loop. We take EINSTein as simulation experiment platform for implementing the agent-based simulation model. By this simulation, the warfare result can be illustrated by Fig. 5, which shows the damages to targets.

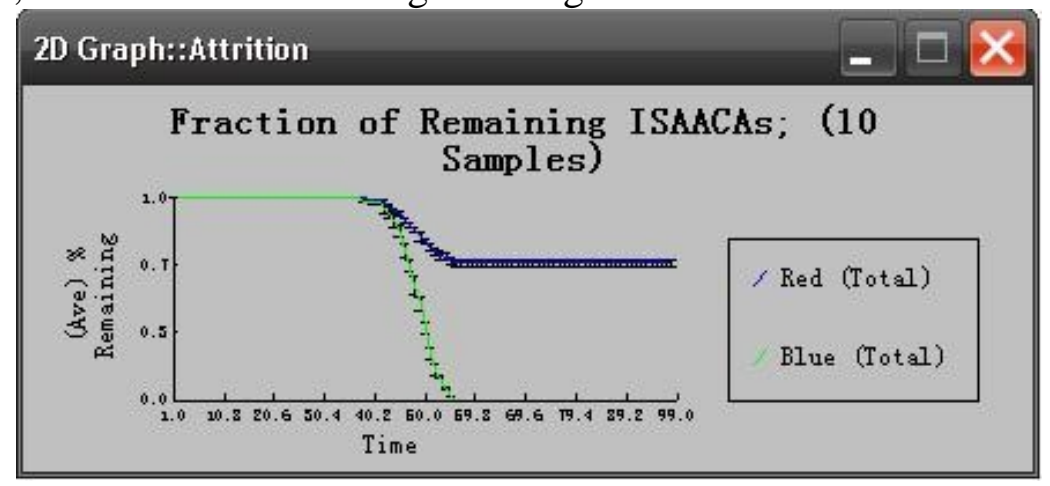

Fig.5 Warfare result on damages to targets in simulation.

\section{Conclusions}

For some time now target-centric warfare has attracted the interest of researchers far beyond military science. According to the demands of modeling and simulation for target-centric warfare, agent-based model is presented in this paper. The approach can make intelligent simulation more practical.

\section{Acknowledgements}

This work is supported by the National Natural Science Foundation of China under Grant 61473311 and Equipment Projects of PLA.

\section{References}

[1] U.S. Army War College. U.S. V Corps and 3rd Infantry Division (Mechanized) During Operation Iraq Freedom Combat Operations (Mar-Apr 2003) Volume I: Operations, (2003).

[2] Xiong Li, Wei Pu. Task Oriented Equipment-level Agent-based Modeling for Emergency Operations. Proceedings of the ICMPMME, 2015, DEStech Publications. p. 47-52.

[3] Ana Lilia La., Tzitziki R., etc., Multi-Agent System for Real Time Planning Using Collaborative Agents, International Journal of Intelligence Science, 4(2014), p. 91-103.

[4] Olcay Akman, Dan Hrozencik. Using an Agent-based Model to Study the Effect of Reproductive Skew on Mongoose Populations, Adaptive Behavior, Vol. 24(3) (2016), p. 160-167.

[5] Xiong Li, Zhiming Dong. Platform-Level Distributed Warfare Model Based on Multi-Agent System Framework, Defence Science Journal, Vol. 62(3) (2012), p. 180-186.

[6] Xiong Li. Agent-based Warfare Modeling (National Defense Industry Press, Beijing, China 2013).

[7] Victor Lesser. Autonomous Agents and Multi-Agent Systems (Kluwer, Boston, USA 1998).

[8] Zhongzhi Shi, Jianhua Zhang, etc., A Cognitive Model for Multi-Agent Collaboration, International Journal of Intelligence Science, 4(2014), p. 1-6.

[9] Clay Wilson. Network Centric Warfare: Background and Oversight Issues for Congress, (2004). 